\title{
Doxorubicin Caused Apoptosis of Mesenchymal Stem Cells via p38, JNK and p53 Pathway
}

\author{
Fan Yang ${ }^{\mathrm{a}, \mathrm{c}}$ Hongyang Chen ${ }^{\mathrm{a}, \mathrm{c}}$ Yanju Liu ${ }^{\mathrm{a}}$ Kun Yin ${ }^{\mathrm{a}}$ Yang Wang ${ }^{\mathrm{a}}$ Xingda $\mathrm{Li}^{\mathrm{a}}$ \\ Guohui Wang ${ }^{a}$ Siyue Wang ${ }^{a}$ Xueying Tan ${ }^{a}$ Chaoqian Xua Yanjie Lua ${ }^{a}$ benzhi Caia
}

aDepartment of Pharmacology, State-Province Key Laboratories of Biomedicine- Pharmaceutics of China, Harbin Medical University, 'Institute of Cardiovascular Research, Harbin Medical University, Harbin, China; 'The first two authors made equal contribution to this work

\section{Key Words}

Doxorubicin • Apoptosis • Bone marrow-derived mesenchymal stem cells $\bullet$ p53 • ROS

\begin{abstract}
Background/Aims: Doxorubicin is a widely used chemotherapeutic agent, but its clinical use is restricted because of a high risk of cardiotoxicity. Bone marrow-derived mesenchymal stem cells (BMSCs) may repair ischaemically damaged myocardium through transdifferentiation and paracrine action. The aim of this study is to investigate if doxorubicin causes the apoptosis of BMSCs and in turn impairs its healing ability. Methods: BMSCs were exposed to doxorubicin, and cell apoptosis was determined by western blot and stainings. Results: Doxorubicin reduced the survival ratio and caused the apoptosis of BMSCs, with the increase of intracellular ROS level and depolarization of mitochondrial membrane potential. The ROS scavenger NAC abrogated these consequences. Moreover, doxorubicin markedly activated phosphorylated ERK, p38 and JNK proteins in BMSCs. The specific inhibitors for p38 (SB203580) and JNK (SP600125) may abolish doxorubicin-induced apoptosis of BMSCs but the specific ERK inhibitor (PD98059) not, indicating p38 and JNK activation contribute to BMSCs apoptosis. Also, the phosphorylated and total p53 proteins were increased in doxorubicin-treated BMSCs. Proapoptotic cleaved caspases-3 was upregulated and antiapoptotic $\mathrm{BCl}-2$ protein was reduced in doxorubicintreated BMSCs. At last, ELISA assay showed that doxorubicin treatment reduced the VEGF and IGF-1 released by BMSCs. Conclusion: Taken together, doxorubicin caused BMSCs apoptosis associated with $\mathrm{p} 38$, JNK and p53 pathways.
\end{abstract}




\section{Introduction}

Doxorubicin (DOX) is a widely used chemotherapeutic agent and effective in treating hematologic and solid tumor malignancies, including leukemia, breast cancer, lung carcinoma, kidney cancer, etc $[1,2]$. Despite its high efficacy, the clinical application of doxorubicin was greatly restricted due to its multiple side effects [3, 4]. Especially in heart tissues, doxorubicin may cause dilated cardiomyopathy, congestive heart failure and even cardiac sudden death [5-7]. The pathological mechanisms of doxorubicin cardiotoxicity are associated with oxidative stress and cardiomyocyte apoptosis [8, 9]. AKT, PI3K, calcineurin, p38 and JNK signal pathways have been shown responsible for doxorubicin toxicity [10]. In addition, p53, p21 and G2/M cell cycle arrest were also involved in doxorubicin-induced ROS generation and apoptosis [11]. Recently, doxorubicin-induced autophagy was shown contributed to doxorubicin cardiotoxicity via decreasing GATA-4 protein expression [12]. Though many advances have been made in the understanding of doxorubicin toxicity, the complete mechanism has not been elucidated yet.

As one type of adult stem cells, mesenchymal stem cells derived from bone marrow (BMSCs) are capable to differentiate into cardiomyocytes and vascular cells, and in turn regenerate the damaged myocardium and improve heart functions $[13,14]$. Moreover, BMSCs also can secret some cytokines including VEGF, IGF-1, etc, and even microRNAs to protect the heart against ischemic or hypoxic injury [15]. Thus, the dysfunction or apoptosis of BMSCs caused by hypoxia, ischemia or other pathological factors will weaken the regenerative ability of BMSCs and promote the development of cardiovascular diseases [16]. In vivo animal studies also displayed only a small number of BMSCs survive and then differentiate into cardiac cells under ischemic and inflammatory condition [14,17]. Here, we hypothesize that doxorubicin causes the apoptosis or dysfunction of BMSCs which contributes to, at least partially doxorubicin-related cardiovascular disorders. Our study uncovered that doxorubicin promoted ROS generation, activated p38, JNK and p53 pathways, and caused mitochondrial membrane potential depolarization and caspase- 3 activation, which consequently triggered the apoptosis and dysfunction of BMSCs.

\section{Materials and Methods}

\section{Animals}

The Male Sprague-Dawley (SD) rats (about $100 \mathrm{~g}$ ) were purchased from the experimental animal center of Harbin medical university, and all experimental procedures were approved by the Ethic Committee of Harbin Medical University. In addition, all animal protocols were carried out in accordance to the Guide for the Care and Use of Laboratory Animals published by the US National Institute of Health.

Reagent

Doxorubicin was purchased from Pfizer Italia Srl (Nerviano, Italy). All chemicals were purchased from Sigma (St, Louis. Mo. USA) unless mentioned otherwise. AO/EB was brought from Roche Company (Penzberg, Germany). Live/Dead® assay kit was purchased from Invitrogen (Invitrogen, USA). Mesenchymal Stem Cell Culture Medium was brought from Stem Cell Technologies Inc (Stem Cell, Canada). EnzymeLinked Immunosorbent Assay (ELISA) kit was obtained from (WuHan Boster Co., Ltd., China). JC-1 probe and Reactive Oxygen Species (ROS) detection assay kit were purchased from Beyotime (Beyotime, China).

Bone marrow mesenchymal stem cells

BMSCs derived from young SD rats were primarily cultured just as described previously [18]. In brief, BMSCs were acutely isolated from rat bone marrow and then were cultured with stem cell medium (Stem Cell, Canada) supplemented with $20 \%$ special serum at $37{ }^{\circ} \mathrm{C}$. The culture medium was changed every $2-3$ days. All experiments were performed using cultured BMSCs from third passage. 


\section{Acridine Orange/Ethidium Bromide (AO/EB) Staining}

BMSCs apoptosis was detected using acridine orange/ethidium bromide (AO/EB) staining [19]. The cultured BMSCs were treated with different concentrations of doxorubicin for $48 \mathrm{~h}$. In order to label the apoptotic cells, $10 \mu \mathrm{L}$ prepared AO/EB working solution (100 $\mu \mathrm{g} / \mathrm{mL}$ AO and $100 \mu \mathrm{g} / \mathrm{mL}$ EB in PBS) was added to the cells for $5 \mathrm{~min}$. Then, the cultured cells were observed using an inverted fluorescence microscope (Eclipse TE300, Nikon, Japan).

\section{Live/Dead viability staining}

To measure the viability of doxorubicin-treated BMSCs, we used the Live/Dead $\AA$ assay kit (Invitrogen) to determine the intracellular esterase activity and plasma membrane integrity. In brief, the cultured BMSCs were incubated with different concentrations of doxorubicin for $48 \mathrm{~h}$. Then the BMSCs were stained with the Live/Dead reagent (Ethidium Homodimer and Calcein-AM) and incubated at $37^{\circ} \mathrm{C}$ for $30 \mathrm{~min}$. The viability of BMSCs was then analyzed under a fluorescence microscope (Eclipse TE300, Nikon, Japan).

\section{TUNEL assay}

Effects of doxorubicin on BMSCs apoptosis were determined using Terminal deoxynucleotidyl transferase dUTP nick end labeling (TUNEL) assay as described previously [19]. The cultured BMSCs were fixed with $4 \%$ paraformaldehyde for $1 \mathrm{~h}$ at room temperature, blocked and then permeabilized in $0.1 \%$ Triton X-100. The fixed cells were exposed to freshly prepared TUNEL reaction mixture for $1 \mathrm{~h}$ in a dark room. The cells were then washed with PBS and observed by a fluorescence microscope (Eclipse TE300, Nikon, Japan).

\section{Measurement of Reactive Oxygen Species (ROS)}

To quantify intracellular ROS level, we used 2,7-dichlorodihydrofluorescein diacetate (H2DCF-DA) probe. The method to measure ROS generation was just as previously described [20]. Briefly, cultured BMSCs were incubated with different concentrations of doxorubicin. After rinsing three times with PBS, the cells were loaded with H2DCF-DA $10 \mu \mathrm{M}$ for $20 \mathrm{~min}$ at $37^{\circ} \mathrm{C}$. After fixed in $4 \%$ paraformaldehyde,and washing with PBS, the cells were stained with DAPI and were then imaged with a fluorescence microscope (Eclipse TE300, Nikon, Japan).

Mitochondrial membrane potential (MMP) assay

Mitochondrial membrane potential (MMP) was determined using JC-1 staining. In brief, after treatment with doxorubicin for $48 \mathrm{~h}$, cultured BMSCs were exposed to JC-1 probe, and then the cultured medium of BMSCs was removed. After the cells were rinsed in PBS, the cultured cells were imaged with a fluorescence microscope (Eclipse TE300, Nikon, Japan).

\section{ELISA assay}

ELISA was used to determine the level of VEGF and IGF-1 in the culture medium of BMSCs. In brief, cultured BMSCs were exposed to different concentrations of doxorubicin. After $48 \mathrm{~h}$, the culture medium of BMSCs was harvested and centrifuged, and the levels of VEGF and IGF- 1 in the supernatant were measured using VEGF and IGF-1 ELISA kits according to the manufacturer's instruction.

\section{Western Blot}

After extracted protein from cultured BMSCs, the determination of protein concentration was achieved by the BCA protein assay. The fractionated proteins were analyzed by SDS-PAGE (10\%-15\% polyacrylamide gels) and transferred to PVDF membrane (Millipore, Bedford, MA). After blocked with $5 \%$ non-fat milk for 1 $\mathrm{h}$, the membranes were then incubated with primary antibodies for phospho-ERK1/2 (Abcam, UK), ERK1/2 (Abcam, UK), phospho-JNK (Abcam, UK), JNK (Abcam, UK), phospho-p38 (Abcam, UK), p38 (Abcam, UK), Bax (Santa Cruz, USA), Bcl-2 (Santa Cruz, USA), phospho-p53 (Santa Cruz, USA), p53 (Santa Cruz, USA), caspase-3 (Santa Cruz, USA), cleaved caspase-3 (Santa Cruz, USA) at $4{ }^{\circ} \mathrm{C}$ overnight. After rinsing in PBS, the membranes were incubated with mouse or rabbit secondary antibodies for $1 \mathrm{~h}$ at room temperature. The blotting bands were quantified by measuring the band intensity (area×OD) using Odyssey v1.2 software and normalizing to GAPDH (Santa Cruz, USA) as an internal control. 


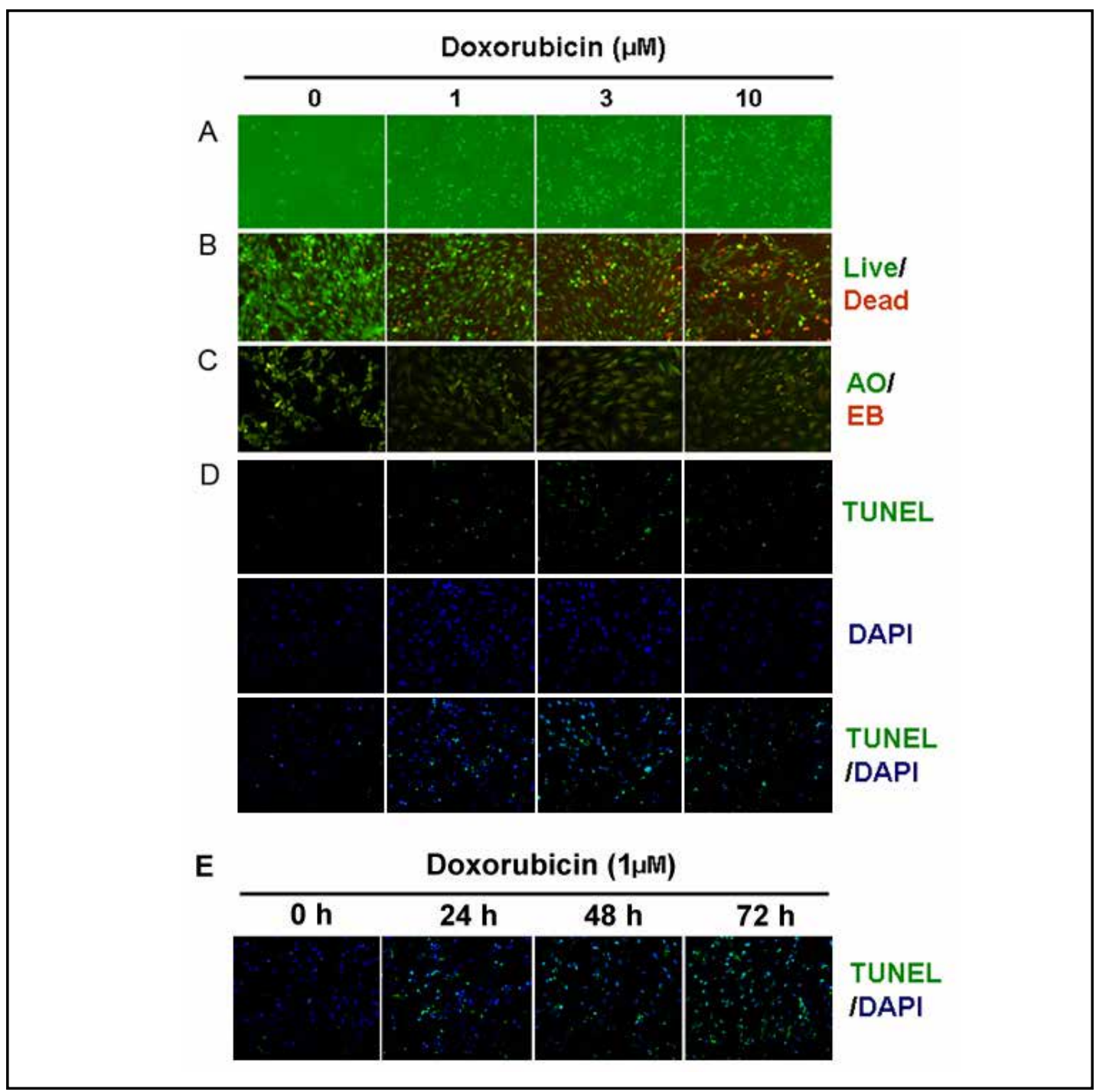

Fig. 1. Doxorubicin induced apoptotic morphological changes and decreased BMSCs viability. (A) Apoptotic morphological appearance such as cell shrinkage was observed in BMSCs after exposure to different concentrations of doxorubicin $(1,3,10 \mu \mathrm{M})$ for $48 \mathrm{~h}$. (B) Doxorubicin-treated BMSCs exhibited a considerable reduction of living cells compared with control cells by Live/Dead staining. (C) The effects of doxorubicin 1,3 and $10 \mu \mathrm{M}$ on the apoptosis of BMSCs by AO/EB staining. (D) Effects of different concentration of doxorubicin on the number of apoptotic BMSCs by TUNEL staining. (E) Doxorubicin caused the augment of the number of apoptotic BMSCs with time increasing.

\section{Statistical Analysis}

Data was expressed as mean \pm S.E.M. The Student's $t$ test was used to determine the statistical significance of differences between two groups, and ANOVA was used to determine the significance of differences among groups. The $\mathrm{p}<0.05$ indicates a statistical significance. All statistical analysis was performed using the Graphpad Prism 4.0 software.

\section{Results}

Doxorubicin induced the apoptosis of BMSCs

Firstly, doxorubicin-induced damage of BMSCs was determined using AO/EB, Live/ Dead and TUNEL staining. As shown in Figure 1A, exposure to doxorubicin 1, 3, $10 \mu \mathrm{M}$ 


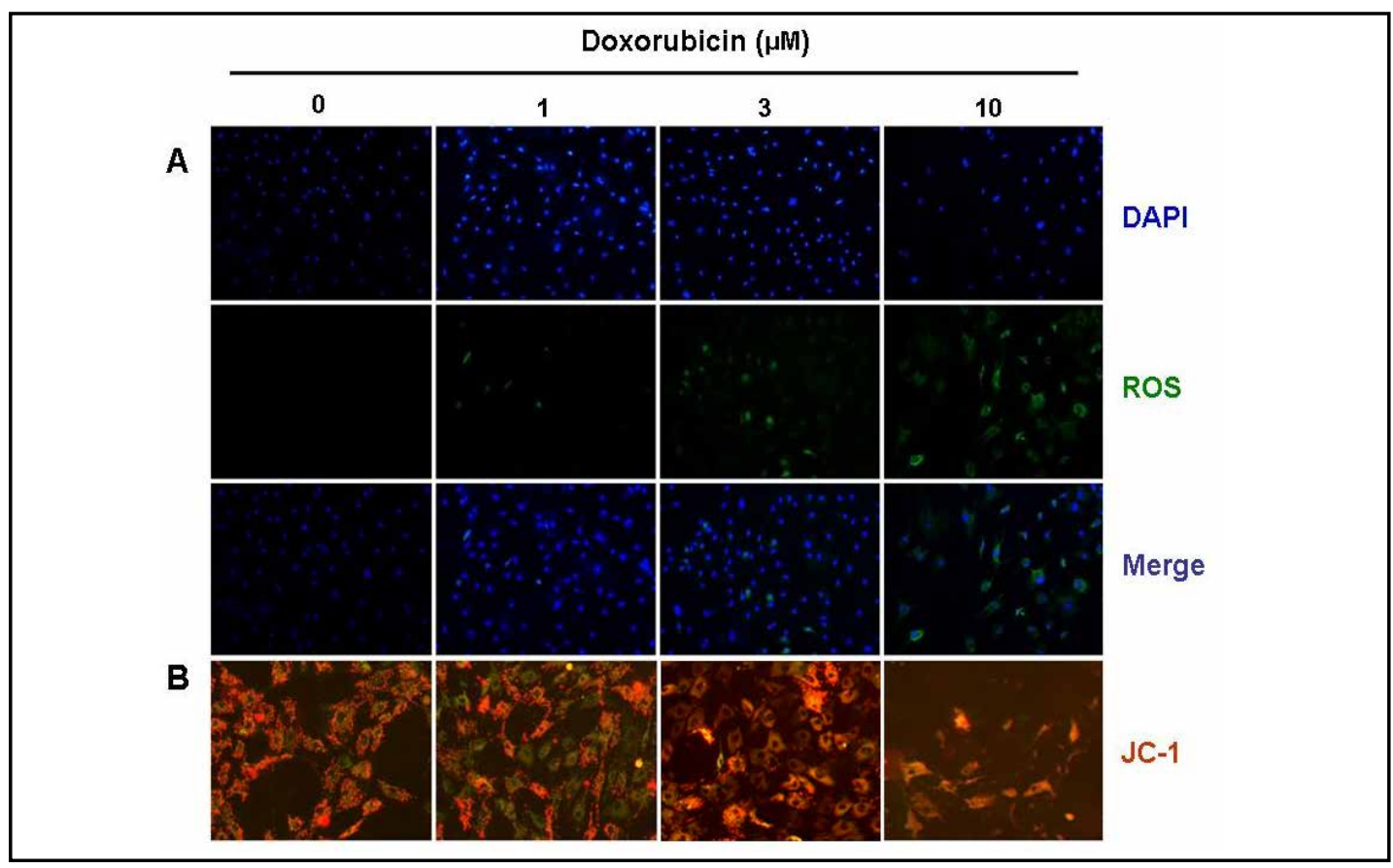

Fig. 2. Doxorubicin increased intracellular ROS level and disrupted mitochondrial membrane potential in BMSCs. (A) H2DCF-DA staining displayed a increase of intracellular ROS production in BMSCs following treatment with doxorubicin 1, 3 and $10 \mu \mathrm{M}$ for $48 \mathrm{~h}$. (B) Exposure to doxorubicin 1, 3 and $10 \mu \mathrm{M}$ for $48 \mathrm{~h}$ resulted in the depolarization of mitochondrial membrane potential of BMSCs by JC- 1 staining.

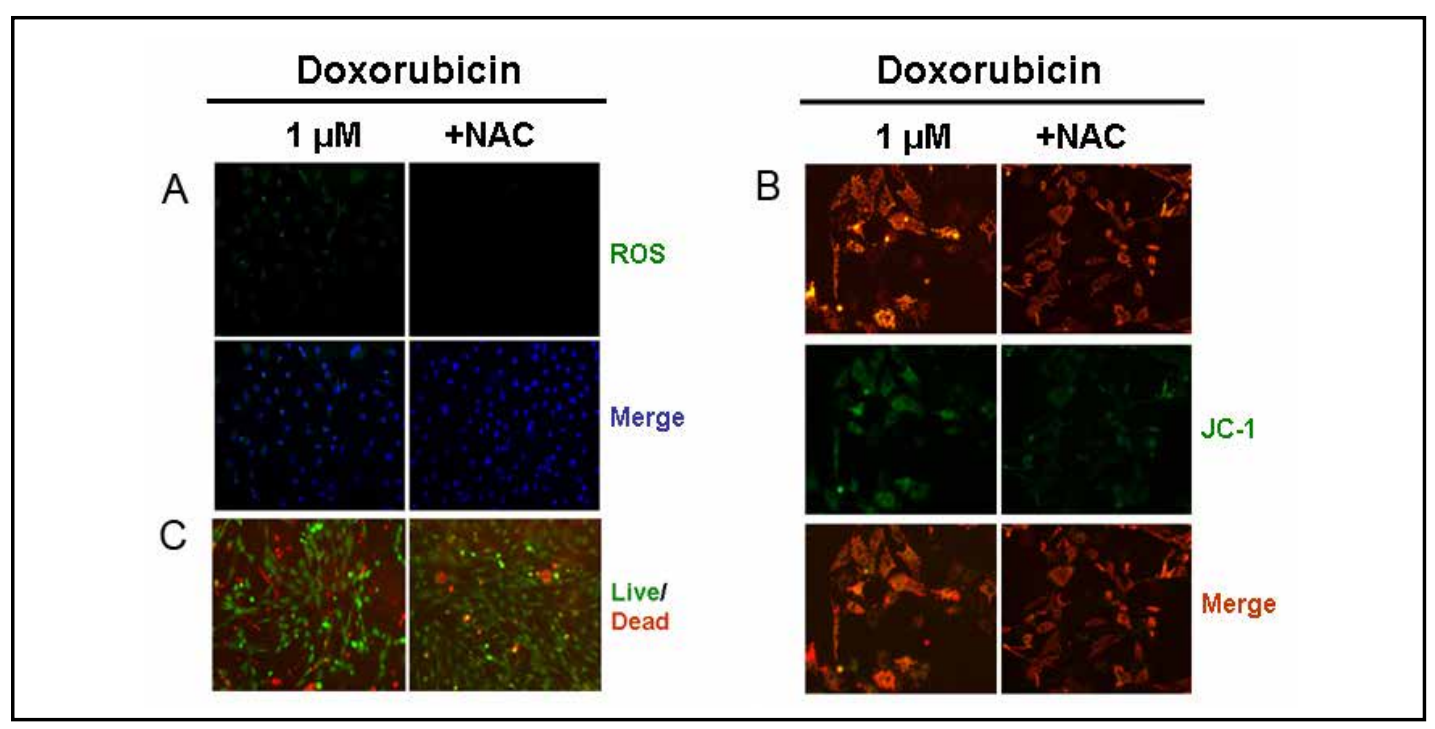

Fig. 3. Antioxidant reversed doxorubicin-induced the apoptosis of BMSCs. (A) Increased ROS level in doxorubicin-treated BMSCs was reversed after treatment with antioxidant NAC by H2DCF-DA staining. (B) Pretreatment with NAC also attenuated the depolarization of mitochondrial membrane potential in BMSCs induced by doxorubicin $1 \mu \mathrm{M}$. (C) Doxorubicin-induced the decrease of BMSCs viability was also reversed by NAC. Data are for three independent experiments.

resulted in distinct morphological changes such as cellular shrinkage of BMSCs. Figure 1B demonstrated that the percentage of living BMSCs with green staining was significantly reduced after treatment with doxorubicin for $48 \mathrm{~h}$, and the dead BMSCs with red, orange or yellow staining were obviously increased, suggesting a lack of membrane integrity. Then, 
A

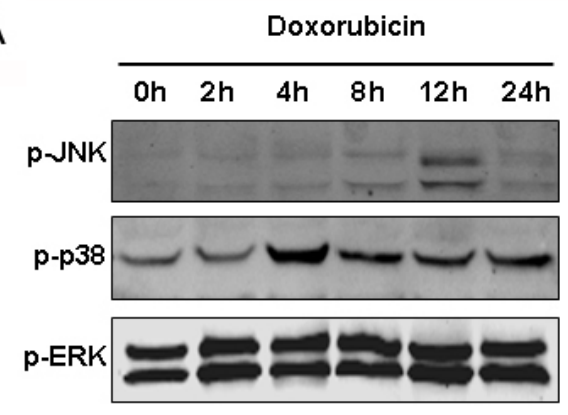

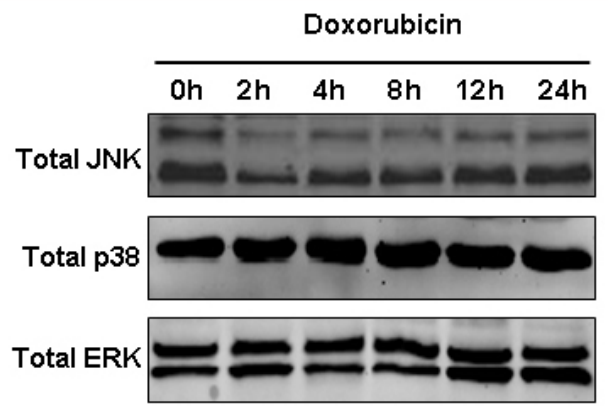

Doxorubicin

B

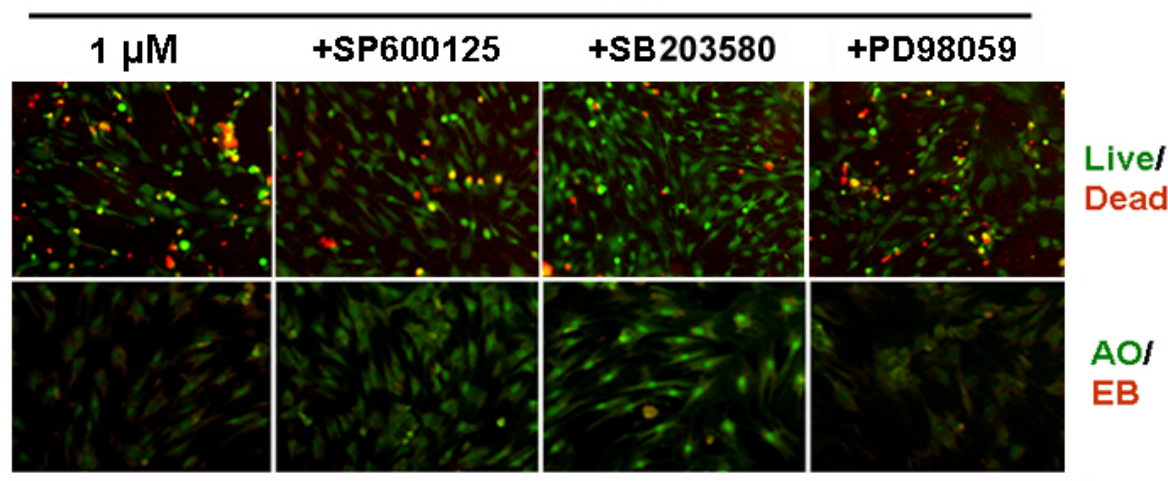

Fig. 4. JNK and p38 signal contributed to doxorubicin-induced BMSCs apoptosis. (A) The expression of total and phosphorylated ERK, p38 and JNK was measured by western blot in BMSCs treated with doxorubicin $1 \mu \mathrm{M}$ at $0,2,4,8,12$ and $24 \mathrm{~h}$. The phosphorylation of ERK, p38 and JNK was significantly increased after doxorubicin $1 \mu \mathrm{M}$ treatment. (B) Doxorubicin-induced the apoptosis of BMSCs was abrogated by p38 and JNK specific inhibitors, but ERK specific inhibitors did not reverse the apoptosis of BMSCs induced by doxorubicin.

AO/EB staining showed that the apoptotic morphological changes in the nucleus and the cytoplasm were found in BMSCs after treatment with doxorubicin 1, 3 and $10 \mu \mathrm{M}$ (Fig. 1C). Furthermore, TUNEL staining was used to evaluate the proapoptotic effects of doxorubicin on BMSCs, and the result displayed the number of apoptotic BMSCs was increased in the presence of doxorubicin, which is associated with the concentration and exposure time of doxorubicin (Fig. 1D-1E).

\section{Doxorubicin enhanced ROS and depolarized mitochondrial membrane potential}

Growing evidence shows that excessive ROS (Reactive Oxygen Species) production and subsequent mitochondrial membrane damage are responsible for cellular apoptosis [21]. Thus, we assessed if intracellular ROS production and mitochondrial membrane integrity of BMSCs were influenced by doxorubicin. As displayed in Figure 2A, after exposing to doxorubicin 1, 3, $10 \mu \mathrm{M}$ for $48 \mathrm{~h}$, BMSCs exhibited a gradual increase of intracellular ROS generation as illuminated by enhanced green staining in the cytoplasm. Meanwhile, the disruption of mitochondrial membrane potential was observed in BMSCs after doxorubicin treatment (Fig. 2B). These results suggest that doxorubicin not only induces the BMSCs to produce ROS, but also causes the loss of mitochondrial membrane potential.

Antioxidant reversed doxorubicin-induced the apoptosis of BMSCs

To further confirm whether ROS is required for the induction of BMSCs apoptosis, ROS scavenger acetylcysteine (NAC) was used in the further assays. Figure $3 \mathrm{~A}$ showed that NAC 


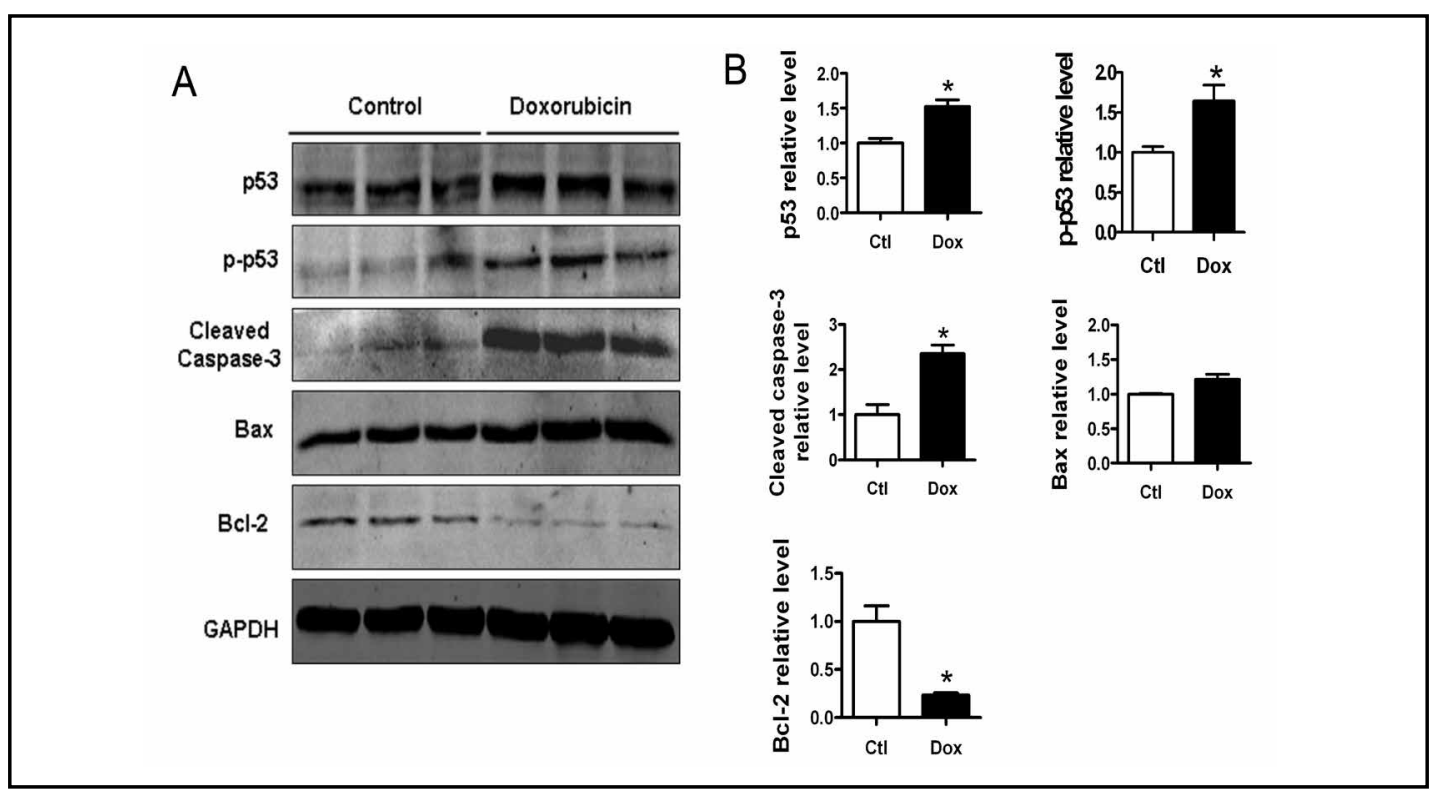

Fig. 5. Doxorubicin activated p53 and upregulated the expression of apoptotic proteins in BMSCs. (A) Doxorubicin $1 \mu \mathrm{M}$ resulted in an increase of the expression of phosphorylated p53 and total p53 in BMSCs. (B) Effects of doxorubicin on the Bax and Bcl-2 proteins in BMSCs. Doxorubicin obviously downregulated Bcl-2 protein. The augmented influences of doxorubicin on the expression of cleaved caspase- 3 in BMSCs. Data are expressed as mean \pm S.E.M for three independent experiments. ${ }^{*} \mathrm{p}<0.05$ versus Control.

attenuated the increase of ROS in BMSCs induced by doxorubicin. Meanwhile, doxorubicininduced the depolarization of mitochondrial membrane potential was also blocked by NAC pretreatment (Fig. 3B). The Live/Dead staining showed that the increase of apoptotic BMSCs induced by doxorubicin was abrogated by NAC (Fig. 3C). It suggests that doxorubicin-induced apoptosis of BMSCs involves ROS-mediated mitochondrial membrane depolarization.

\section{JNK and 38 is involved in doxorubicin-induced the apoptosis of BMSCs}

The mitogen activated protein kinases (MAPKs) signal pathway plays an important role in cellular apoptosis, especially in response to oxidant stress [22]. Thus, we hypothesized the proapoptotic effect of doxorubicin on BMSCs is attributed to the activation of ERK, p38 and JNK. As shown in Figure 4A, doxorubicin $1 \mu \mathrm{M}$ increased the expression of the phosphorylation of ERK, p38 and JNK. The expression of phosphorylated ERK was increased by doxorubicin from $2 \mathrm{~h}$, and the phosphorylation of p38 and JNK was enhanced from $4 \mathrm{~h}$ after doxorubicin treatment.

Furthermore, three MAPKs specific inhibitors were used to determine the role of ERK, p38 and JNK activation in doxorubicin-induced apoptosis of BMSC. The results showed that the specific JNK inhibitor SP600125 $(10 \mu \mathrm{M})$ and p38 MAKP inhibitor SB203580 (5 $\mu \mathrm{M})$ attenuated doxorubicin-induced the apoptosis of BMSCs and enhanced cellular viability of BMSCs, while the specific ERK inhibitor PD98059 $(25 \mu \mathrm{M})$ did not produce any effects on the apoptosis of BMSCs induced by doxorubicin (Fig. 4B). These results indicate that doxorubicin-induced the apoptosis of BMSCs is triggered by the activation of p38 and JNK but not ERK signal.

Doxorubicin increased the expression of proapoptotic proteins in BMSCs

Activation of p53 and other proapoptotic proteins have been shown to mediate doxorubicin-induced apoptosis of cardiomyocytes [22]. Herein, we next evaluated the expression of p53, phosphorylated p53 (p-p53), cleaved caspase-3, Bax, Bcl-2 proteins on doxorubicin-treated BMSCs. The result showed that exposure to doxorubicin $1 \mu \mathrm{M}$ obviously increased the expression of both p-p53 and total p53 expression in BMSCs (Fig. 5A). Further 

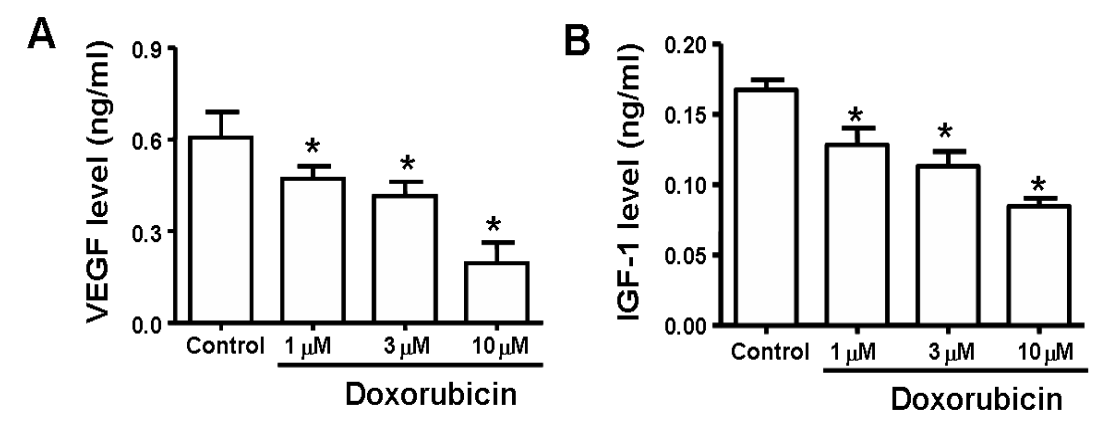

Fig. 6. Doxorubicin inhibited the release of VEGF and IGF-1 by BMSCs. (A) The decrease of VEGF level in the culture medium was induced by doxorubicin 1,3 and $10 \mu \mathrm{M}$ in BMSCs. (B) Also, doxorubicin administration reduced the IGF-1 level in the culture medium of BMSCs. ${ }^{*} \mathrm{p}<0.05$ versus Control.

investigation demonstrated that antiapoptotic protein Bcl-2 was downregulated in BMSCs after doxorubicin treatment (Fig. 5B). We also evaluated the influences of doxorubicin on the expression of cleaved caspase- 3 in BMSCs. Figure 5B showed that doxorubicin $1 \mu \mathrm{M}$ resulted in an increase of the expression of cleaved caspase-3.

Doxorubicin impaired the paracrine function of BMSCs

We further studied if doxorubicin-induced apoptosis of BMSCs resulted in the dysfunctions of BMSCs. As displayed in Figure 6A, the level of VEGF released by BMSCs in culture medium was dose-dependently reduced in the presence of doxorubicin. Similarly, BMSCs-secreted another important factor IGF-1 level was also decreased in the medium of doxorubicin-treated BMSCs (Fig. 6B). It suggests that exposure to doxorubicin impairs the paracrine action and results in the dysfunction of BMSCs.

\section{Discussion}

The present study firstly revealed that doxorubicin caused the increase of ROS and depolarization of mitochondrial membrane potential, as well as the activation of p38, JNK and p53 signal, and in turn trigger the apoptosis and dysfunction of BMSCs. These findings provide evidence for a better understanding of the mechanisms underlying doxorubicinrelated cardiovascular toxicity.

In clinics, doxorubicin is one of the most effective chemotherapeutic drug for leukemia, Hodgkin's lymphoma, and cancers of the bladder, breast, stomach, lung, ovaries and others $[1,2]$. Despite high efficiency in the therapeutics of these tumors, doxorubicin's application was often accompanied by some unavoidable adverse effects such as cardiovascular toxicity $[4,7]$. Some studies have revealed its potential mechanisms including the disruption of energy metabolism, apoptosis and increased $\operatorname{ROS}$ and mitochondrial injury $[3,4,8,9,22]$. However, the underlying mechanism of doxorubicin toxicity on cardiovascular system has not been fully understood yet.

BMSCs play a supporting role in improving cardiovascular functions $[13,14]$. Thus we hypothesized that doxorubicin-induced BMSCs apoptosis might contribute to its cardiovascular toxicity. Live/Dead staining showed that doxorubicin inhibited the viability of BMSCs. In order to demonstrate if doxorubicin produces proapoptotic toxicity on BMSCs, we used AO/EB and TUNEL staining to assess BMSCs apoptosis, and the results showed that BMSCs developed apoptosis in the presence of doxorubicin 1, 3 and $10 \mu \mathrm{M}$ which is relevant to clinical conditions. The ROS and JC-1 staining were further used to explore the potential mechanisms of doxorubicin-induced apoptosis of BMSCs. The results showed that the ROS of BMSCs treated by doxorubicin was markedly increased, along with the loss of mitochondrial 
membrane potential. Furthermore, the ROS inhibitor NAC can abolish the apoptosis of BMSCs induced by doxorubicin, indicating doxorubicin-induced ROS is involved in BMSCs apoptosis.

MAPKs pathway plays a major role in signal transduction and mediates cellular proliferation, differentiation, inflammation and apoptosis, which consists of extracellular signal-regulated kinase (ERK), p38 and Jun N-terminal kinase (JNK) [23]. Previous studies reported that ROS activated JNK and p38 MAPK which takes part in cellular apoptosis, while the activation of ERK by growth factors and pathological stimuli confers anti-oxidative effect and cellular protection $[24,25]$. Therefore we detected the changes of total and phosphorylation of ERK, p38 and JNK in BMSCs after treatment with doxorubicin, and the results showed that doxorubicin treatment increased ERK, p38 and JNK phosphorylation expression. Consistently, previous studies also reported that p38 and JNK were also activated in doxorubicin-treated cardiomyocytes [21,26]. The phosphorylated ERK was seldom observed activated by doxorubicin in hearts in prior reports. However, we found doxorubicin also enhanced the expression of phosphorylated ERK. Nevertheless, the specific ERK inhibitor could not reverse the apoptosis of BMSCs treatment, indicating the activation of ERK does not contribute to the apoptosis of BMSCs [21]. The specific inhibitors for p38 and JNK can attenuate the apoptosis of BMSCs, indicating that p38 and JNK activation are responsible for doxorubicin-induced apoptosis in BMSCs.

In addition, we investigated apoptosis-associated genes which might contribute to doxorubicin-induced apoptosis of BMSCs. Firstly we analyzed the expressions of total p53 and p-p53 proteins, which were the most extensively characterized tumor suppressor protein and regulated metabolism, genomic stability, senescence and apoptosis [27]. In the present study, we found that doxorubicin obviously increased the expression of p-p53 in BMSCs. In agreement, previous studies also demonstrated that doxorubicin-induced p53 activation in tumor cells was mediated by ROS generation [28]. These activated signals subsequently evoked downstream genes such as Bax and Bcl-2 to cause cellular apoptosis [29]. Meanwhile, Bcl-2 family was also a downstream component of p38 and JNK signal pathway [30]. Therefore we detected the expression of Bax and Bcl-2 proteins and found that doxorubicin-induced apoptosis of BMSCs was accompanied by the downregulation of antiapoptotic protein Bcl-2. These results implied that ROS-mediated activation of p38, JNK and $\mathrm{p} 53$ pathways were involved in doxorubicin-induced BMSCs apoptosis $[26,31,32]$. Then, we further found that doxorubicin upregulated the expression of cleaved caspase- 3 protein in BMSCs. This finding further confirmed the evidence of doxorubicin-induced apoptosis of BMSCs. Finally, we employed ELISA assay to detect the effects of doxorubicin on paracrine functions of BMSCs, and found that doxorubicin could inhibit the ability of BMSCs to secret VEGF and IGF-1 and reduce the VEGF and IGF-1 level in the medium, suggesting that the apoptosis of BMSCs could decrease the healing ability of BMSCs in injured hearts.

In summary, our data uncovered that doxorubicin could cause the apoptosis and dysfunction of BMSCs mainly through the increased ROS and the loss of mitochondrial membrane potential, as well as the activation of p38, JNK and p53, which cooperatively triggers apoptosis and dysfunction of BMSCs. This study helps us toward a better understanding of potential mechanisms of doxorubicin toxicity, and provides new strategy for the prevention of doxorubicin-associated heart disorders.

\section{Conflicts of Interest}

All authors have no conflicts of interest.

\section{Acknowledgements}

This study was supported by the National Basic Research Program of China (973 Program 2013CB531104), the Natural Science Foundation of China (81170096), the Program for New Century Excellent Talents In Heilongjiang Provincial University (1252-NCET-013). 


\section{References}

1 Chahinian AP, Antman K, Goutsou M, Corson JM, Suzuki Y, Modeas C, Herndon JE, 2nd, Aisner J, Ellison $\mathrm{RR}$, Leone L, et al.: Randomized phase ii trial of cisplatin with mitomycin or doxorubicin for malignant mesothelioma by the cancer and leukemia group b. J Clin Oncol 1993;11:1559-1565.

2 Bielack SS, Erttmann R, Kempf-Bielack B, Winkler K: Impact of scheduling on toxicity and clinical efficacy of doxorubicin: What do we know in the mid-nineties? Eur J Cancer 1996;32A:1652-1660.

3 Zhang S, Liu X, Bawa-Khalfe T, Lu LS, Lyu YL, Liu LF, Yeh ET: Identification of the molecular basis of doxorubicin-induced cardiotoxicity. Nat Med 2012;18:1639-1642.

4 Horie T, Ono K, Nishi H, Nagao K, Kinoshita M, Watanabe S, Kuwabara Y, Nakashima Y, Takanabe-Mori R, Nishi E, Hasegawa K, Kita T, Kimura T: Acute doxorubicin cardiotoxicity is associated with mir-146ainduced inhibition of the neuregulin-erbb pathway. Cardiovasc Res 2010;87:656-664.

5 Wojnowski L, Kulle B, Schirmer M, Schluter G, Schmidt A, Rosenberger A, Vonhof S, Bickeboller H, Toliat MR, Suk EK, Tzvetkov M, Kruger A, Seifert S, Kloess M, Hahn H, Loeffler M, Nurnberg P, Pfreundschuh M, Trumper L, Brockmoller J, Hasenfuss G: Nad(p)h oxidase and multidrug resistance protein genetic polymorphisms are associated with doxorubicin-induced cardiotoxicity. Circulation 2005;112:3754-3762.

6 Batist G, Ramakrishnan G, Rao CS, Chandrasekharan A, Gutheil J, Guthrie T, Shah P, Khojasteh A, Nair MK, Hoelzer K, Tkaczuk K, Park YC, Lee LW: Reduced cardiotoxicity and preserved antitumor efficacy of liposome-encapsulated doxorubicin and cyclophosphamide compared with conventional doxorubicin and cyclophosphamide in a randomized, multicenter trial of metastatic breast cancer. J Clin Oncol 2001;19:1444-1454.

7 Schwartz RG, McKenzie WB, Alexander J, Sager P, D'Souza A, Manatunga A, Schwartz PE, Berger HJ, Setaro J, Surkin L, et al.: Congestive heart failure and left ventricular dysfunction complicating doxorubicin therapy. Seven-year experience using serial radionuclide angiocardiography. Am J Med 1987;82:1109-1118.

8 Fisher PW, Salloum F, Das A, Hyder H, Kukreja RC: Phosphodiesterase-5 inhibition with sildenafil attenuates cardiomyocyte apoptosis and left ventricular dysfunction in a chronic model of doxorubicin cardiotoxicity. Circulation 2005;111:1601-1610.

-9 Fan GC, Zhou X, Wang X, Song G, Qian J, Nicolaou P, Chen G, Ren X, Kranias EG: Heat shock protein 20 interacting with phosphorylated akt reduces doxorubicin-triggered oxidative stress and cardiotoxicity. Circ Res 2008;103:1270-1279.

10 Spallarossa P, Altieri P, Garibaldi S, Ghigliotti G, Barisione C, Manca V, Fabbi P, Ballestrero A, Brunelli C, Barsotti A: Matrix metalloproteinase-2 and -9 are induced differently by doxorubicin in h9c2 cells: The role of map kinases and nad(p)h oxidase. Cardiovasc Res 2006;69:736-745.

-11 Venkatakrishnan CD, Dunsmore K, Wong H, Roy S, Sen CK, Wani A, Zweier JL, Ilangovan G: Hsp27 regulates p53 transcriptional activity in doxorubicin-treated fibroblasts and cardiac h9c2 cells: P21 upregulation and g2/m phase cell cycle arrest. Am J Physiol Heart Circ Physiol 2008;294:H1736-1744.

12 Kobayashi S, Volden P, Timm D, Mao K, Xu X, Liang Q: Transcription factor gata4 inhibits doxorubicininduced autophagy and cardiomyocyte death. J Biol Chem 2010;285:793-804.

13 Silva GV, Litovsky S, Assad JA, Sousa AL, Martin BJ, Vela D, Coulter SC, Lin J, Ober J, Vaughn WK, Branco RV, Oliveira EM, He R, Geng YJ, Willerson JT, Perin EC: Mesenchymal stem cells differentiate into an endothelial phenotype, enhance vascular density, and improve heart function in a canine chronic ischemia model. Circulation 2005;111:150-156.

14 Toma C, Pittenger MF, Cahill KS, Byrne BJ, Kessler PD: Human mesenchymal stem cells differentiate to a cardiomyocyte phenotype in the adult murine heart. Circulation 2002;105:93-98.

15 Xu M, Uemura R, Dai Y, Wang Y, Pasha Z, Ashraf M: In vitro and in vivo effects of bone marrow stem cells on cardiac structure and function. J Mol Cell Cardiol 2007;42:441-448.

16 Barbash IM, Chouraqui P, Baron J, Feinberg MS, Etzion S, Tessone A, Miller L, Guetta E, Zipori D, Kedes LH, Kloner RA, Leor J: Systemic delivery of bone marrow-derived mesenchymal stem cells to the infarcted myocardium: Feasibility, cell migration, and body distribution. Circulation 2003;108:863-868.

17 Khan M, Meduru S, Mohan IK, Kuppusamy ML, Wisel S, Kulkarni A, Rivera BK, Hamlin RL, Kuppusamy P: Hyperbaric oxygenation enhances transplanted cell graft and functional recovery in the infarct heart. J Mol Cell Cardiol 2009;47:275-287. 
18 Benzhi C, Limei Z, Ning W, Jiaqi L, Songling Z, Fanyu M, Hongyu Z, Yanjie L, Jing A, Baofeng Y: Bone marrow mesenchymal stem cells upregulate transient outward potassium currents in postnatal rat ventricular myocytes. J Mol Cell Cardiol 2009;47:41-48.

19 Cai B, Li X, Wang Y, Liu Y, Yang F, Chen H, Yin K, Tan X, Zhu J, Pan Z, Wang B, Lu Y: Apoptosis of bone marrow mesenchymal stem cells caused by homocysteine via activating jnk signal. PLoS One 2013;8:e63561.

20 Cai BZ, Meng FY, Zhu SL, Zhao J, Liu JQ, Liu CJ, Chen N, Ye ML, Li ZY, Ai J, Lu YJ, Yang BF: Arsenic trioxide induces the apoptosis in bone marrow mesenchymal stem cells by intracellular calcium signal and caspase-3 pathways. Toxicol Lett 2010;193:173-178.

21 Ghosh J, Das J, Manna P, Sil PC: The protective role of arjunolic acid against doxorubicin induced intracellular ros dependent jnk-p38 and p53-mediated cardiac apoptosis. Biomaterials 2011;32:48574866.

22 Brantley-Finley C, Lyle CS, Du L, Goodwin ME, Hall T, Szwedo D, Kaushal GP, Chambers TC: The jnk, erk and p53 pathways play distinct roles in apoptosis mediated by the antitumor agents vinblastine, doxorubicin, and etoposide. Biochem Pharmacol 2003;66:459-469.

23 Johnson GL, Lapadat R: Mitogen-activated protein kinase pathways mediated by erk, jnk, and p38 protein kinases. Science 2002;298:1911-1912.

24 Xia Z, Dickens M, Raingeaud J, Davis RJ, Greenberg ME: Opposing effects of erk and jnk-p38 map kinases on apoptosis. Science 1995;270:1326-1331.

25 Fan Y, Chen H, Qiao B, Luo L, Ma H, Li H, Jiang J, Niu D, Yin Z: Opposing effects of erk and p38 map kinases on hela cell apoptosis induced by dipyrithione. Mol Cells 2007;23:30-38.

26 Das J, Ghosh J, Manna P, Sil PC: Taurine suppresses doxorubicin-triggered oxidative stress and cardiac apoptosis in rat via up-regulation of pi3-k/akt and inhibition of p53, p38-jnk. Biochem Pharmacol 2011;81:891-909.

27 Tasdemir E, Maiuri MC, Galluzzi L, Vitale I, Djavaheri-Mergny M, D'Amelio M, Criollo A, Morselli E, Zhu C, Harper F, Nannmark U, Samara C, Pinton P, Vicencio JM, Carnuccio R, Moll UM, Madeo F, Paterlini-Brechot P, Rizzuto R, Szabadkai G, Pierron G, Blomgren K, Tavernarakis N, Codogno P, Cecconi F, Kroemer G: Regulation of autophagy by cytoplasmic p53. Nat Cell Biol 2008;10:676-687.

28 Wang S, Konorev EA, Kotamraju S, Joseph J, Kalivendi S, Kalyanaraman B: Doxorubicin induces apoptosis in normal and tumor cells via distinctly different mechanisms. Intermediacy of $\mathrm{H}_{2} \mathrm{O}_{2}$ - and p53-dependent pathways. J Biol Chem 2004;279:25535-25543.

29 Flores ER, Tsai KY, Crowley D, Sengupta S, Yang A, McKeon F, Jacks T: P63 and p73 are required for p53dependent apoptosis in response to DNA damage. Nature 2002;416:560-564.

-30 De Chiara G, Marcocci ME, Torcia M, Lucibello M, Rosini P, Bonini P, Higashimoto Y, Damonte G, Armirotti A, Amodei S, Palamara AT, Russo T, Garaci E, Cozzolino F: Bcl-2 phosphorylation by p38 mapk: Identification of target sites and biologic consequences. J Biol Chem 2006;281:21353-21361.

- 31 Takekawa M, Adachi M, Nakahata A, Nakayama I, Itoh F, Tsukuda H, Taya Y, Imai K: P53-inducible wip1 phosphatase mediates a negative feedback regulation of p38 mapk-p53 signaling in response to uv radiation. ЕMBO J 2000;19:6517-6526.

32 Poizat C, Puri PL, Bai Y, Kedes L: Phosphorylation-dependent degradation of p300 by doxorubicin-activated p38 mitogen-activated protein kinase in cardiac cells. Mol Cell Biol 2005;25:2673-2687. 